\title{
IncRNA TUG1 regulates human pulmonary microvascular endothelial cell apoptosis via sponging of the miR-9a-5p/BCL2L11 axis in chronic obstructive pulmonary disease
}

\author{
XICHEN CHEN $^{1 *}$, MAO MAO $^{2 *}$, YING SHEN $^{2}$, XUEYONG JIANG ${ }^{2}$ and ZHIFEI YIN ${ }^{2 *}$ \\ ${ }^{1}$ Analysis and Test Center, Nanjing Medical University, Nanjing, Jiangsu 210000; ${ }^{2}$ Rehabilitation Medicine Center, \\ The First Affiliated Hospital of Nanjing Medical University, Nanjing, Jiangsu 210029, P.R. China
}

Received October 10, 2020; Accepted February 18, 2021

DOI: $10.3892 /$ etm.2021.10338

\begin{abstract}
The aim of the present study was to investigate the function of long non-coding RNA taurine-upregulated gene 1 (lncRNA TUG1) in chronic obstructive pulmonary disease and further assess the underlying molecular mechanisms. Flow cytometry analysis was performed to detect cell apoptosis of human pulmonary microvascular endothelial cells (HPMECs) treated with $1 \%$ cigarette smoke extract (CSE). The activity of caspase-3 was measured using a Caspase-3 Activity assay kit and the protein expression of cleaved caspase-3, caspase-3 and Bcl-2 like 11 (BCL2L11) were measured using western blotting. Reverse transcription-quantitative PCR (RT-qPCR) was performed to measure the expression of TUG1 mRNA levels in the treated cells. The association between TUG1, the miR-9a-5p/BCL2L11 axis and with miR-9a-5p were predicted and verified using a dual luciferase reporter assay system. The mRNA expression of miR-9a-5p and BCL2L11, and the transfection efficiency were measured by RT-qPCR. The results showed that CSE induced cell apoptosis and increased lncRNA TUG1 expression in HPMECs. CSE significantly reduced the expression of miR-9a-5p in HPMECs compared with the control group. TUG1-short hairpin RNA relieved cell apoptosis induced by CSE by upregulating miR-9a-5p in HPMECs. The present study predicted and verified that BCL2L11 is a direct target of miR-9a-5p. The mRNA expression of BCL2L11 was increased in HPMECs following CSE treatment compared with the control group. miR-9a-5p mimic and BCL2L11-plasmid markedly increased the expression
\end{abstract}

Correspondence to: Mrs. Zhifei Yin, Rehabilitation Medicine Center, The First Affiliated Hospital of Nanjing Medical University, 300 Guangzhou Road, Nanjing, Jiangsu 210029, P.R. China

E-mail: yinzf101002@163.com

${ }^{*}$ Contributed equally

Key words: chronic obstructive pulmonary disease, long non-coding RNA taurine-upregulated gene 1, microRNA-9a-5p/Bcl-2 like 11 axis of miR-9a-5p and BCL2L11, respectively. miR-9a-5p mimic reversed the increase in cell apoptosis induced by CSE by inhibiting BCL2L11 expression in HPMECs. To conclude, the present study demonstrated that IncRNA TUG1 exerted roles in cell apoptosis induced by CSE through modulating the miR-9a-5p/BCL2L11 axis.

\section{Introduction}

Chronic obstructive pulmonary disease (COPD), which is a progressive degenerative lung disease, is a preventable and treatable disease characterized by incomplete reversibility and airflow limitation, including emphysema and chronic bronchitis (1-3). It is currently the fourth leading cause of death worldwide, just behind ischemic heart disease and cerebrovascular disease, and an increasing number of individuals are diagnosed with the disease (4). Therefore, new and effective treatment strategies targeting COPD are urgently needed. Patients with COPD have difficulty breathing and present with airflow obstruction, which is often accompanied by discomforts, such as coughing and sputum production $(5,6)$. However, the pathogenesis is not yet fully understood. The occurrence of COPD is the result of the combined effects of genetic and environmental pathogenic factors. Cigarette smoke is the most common risk factor for COPD. The rates of respiratory disease, lung function impairment and mortality of smokers are significantly higher compared with non-smokers $(7,8)$. Smoking cessation has been shown to effectively delay the progressive decline of lung function.

Endothelial cells (ECs), as the basic unit of blood vessels, and play key roles in physiological metabolism. Under harmful conditions, the regulatory mechanisms become disrupted, which may be one of the main initiating factors for various diseases $(9,10)$. The pathogenesis of acute lung injury includes alveolitis and tissue recovery, and the vascular response is the central link in the occurrence of inflammation (11). Previous data suggested that abnormal apoptosis serves as a significant factor contributing to the destruction of pulmonary tissue in COPD (12). Both animal and human studies confirmed that endothelial apoptosis plays a key role in the pathogenesis of COPD $(13,14)$. It was reported that human pulmonary microvascular endothelial cells (HPMECs) play important roles in 
the progression of COPD, and the present study further investigated COPD using HPMECs as a model (15).

Long non-coding RNAs (lncRNAs), a class of non-coding RNAs that are $>200$ nucleotides in length, participate in the structure and function of chromatin, and promote a series of biological functions $(16,17)$. An increasing number of studies have suggested that lncRNAs are associated with a number of human diseases, such as cancer, cardiovascular diseases, gynecological diseases and inflammatory diseases $(18,19)$. Studies have suggested that lncRNAs participate in regulating the process of COPD (20). Previously, taurine-upregulated gene 1 (TUG1), one of the most commonly examined lncRNAs, was shown to be upregulated in patients with COPD, and TUG1 knockdown reduced inflammation and airway remodeling in an animal model and reversed inflammation and collagen deposition induced by cigarette smoke extract (CSE) in vitro $(21,22)$. However, the mechanisms and roles of TUG1 in COPD remains unclear.

It was hypothesized that TUG1 may be involved in COPD pathogenesis through regulating EC apoptosis. The present study investigated whether TUG1 played a role in cell apoptosis of HPMECs induced by CSE, and further studied its molecular mechanism in order to provide a more theoretical basis and novel strategies for the treatment of COPD.

\section{Materials and methods}

Cell culture. HPMECs were obtained from the American Type Culture Collection and cultured in DMEM (Gibco; Thermo Fisher Scientific, Inc.) containing 10\% FBS (Gibco; Thermo Fisher Scientific, Inc.) and $1 \%$ penicillin/streptomycin at $5 \% \mathrm{CO}_{2}$ and $37^{\circ} \mathrm{C}$.

CSE preparation. CSE preparation was performed as described previously (23). Smoke from 10 cigarettes (Chengdu Cigarette Factory) was bubbled through DMEM, and this solution was considered $100 \%$ CSE. Then the $100 \%$ CSE solution was frozen and stored at $-80^{\circ} \mathrm{C}$ until required. CSE was diluted with DMEM medium to a final concentration of $1 \%$ for the present study.

Apoptosis assay. HPMECs were treated with 1\% CSE for $24 \mathrm{~h}$, and then collected by trypsinization, washed in PBS and resuspended in $1 \mathrm{X}$ binding buffer at a density of $1 \times 10^{6}$ cells $/ \mathrm{ml}$. A total of $100 \mu \mathrm{l}$ cell suspension was then transferred to a $5 \mathrm{ml}$ tube, and stained with $5 \mu \mathrm{l}$ Annexin V/FITC and PI solution, according to the manufacturer's protocol (BD Biosciences). Stained cells were analyzed by flow cytometry (FCM) using a BD FACSCalibur flow cytometer (BD Biosciences) within $1 \mathrm{~h}$. All the experiments were performed at least three times. Data were analyzed using FlowJo software (version 7.2.4; FlowJo LLC).

Western blot analysis. HPMECs were treated with $1 \%$ CSE for $24 \mathrm{~h}$ and dissolved in RIPA buffer (Beyotime Institute of Biotechnology) after washing in cold PBS. The supernatant was centrifuged at $10,000 \mathrm{x} \mathrm{g}$ at $4^{\circ} \mathrm{C}$ for $15 \mathrm{~min}$ to obtain total protein. Protein concentration was measured using a BCA protein assay kit (Beyotime Institute of Biotechnology).
Equal amounts of protein (40 $\mu \mathrm{g} /$ lane) were separated by $10 \%$ SDS-PAGE. Separated proteins were transferred to PVDF membranes (Thermo Fisher Scientific, Inc.), which were then blocked at room temperature for $1 \mathrm{~h}$ in PBS-Tween-20 (PBS-T) solution containing 5\% non-fat milk. Subsequently, membranes were incubated with cleaved caspase-3 (cat. no. ab32042; 1:1,000; Abcam), caspase-3 (cat. no. ab32351; 1:1,000; Abcam), Bcl-2 like 11 axis (BCL2L11; cat. no. ab32158; 1:1,000; Abcam) or GAPDH (cat. no. sc-47724; 1:500; Santa Cruz Biotechnology, Inc.) antibodies at $4^{\circ} \mathrm{C}$ overnight. Membranes were then washed three times with PBS-T and incubated with the Goat Anti-Mouse IgG Antibody (cat. no. bc-0296G; 1:1,000; BIOSS) or Goat Anti-Rabbit IgG Antibody (cat. no. bc-0295G; 1:1,000; BIOSS) for $1 \mathrm{~h}$ at room temperature. ECL substrate (Thermo Fisher Scientific, Inc.) was used to visualize the protein bands according to the manufacturer's protocol. Band densities were quantified using Gel-Pro Analyzer Densitometry software (version 6.3; Media Cybernetics, Inc.). All the experiments were performed at least three times.

$R N A$ extraction and reverse transcription-quantitative PCR $(R T-q P C R)$. Total cellular RNA was extracted using TRIzol ${ }^{\circledR}$ reagent (Invitrogen; Thermo Fisher Scientific, Inc.) and reverse transcribed into cDNA using a cDNA Synthesis kit according to the manufacturer's protocol (Invitrogen; Thermo Fisher Scientific, Inc.). The relative expression of genes was quantified using a Prism 7000 Real-Time PCR system with SYBR qPCR MasterMix (Vazyme Biotech Co., Ltd.) in accordance with the manufacturer's protocol. Primers were purchased from Sangon Biotech Co., Ltd., and primer sequences were as follows: GAPDH forward, 5'-CTTTGGTATCGTGGAAGGACTC-3' and reverse, 5'-GTAGAGGCAGGGATGATGTTCT-3'; U6 forward, 5'-GCTTCGGCAGCACATATACTAAAAT-3' and reverse, 5'-CGCTTCACGAATTTGCGTGTCAT-3'; lncRNA TUG1 forward, 5'-GACCGTCCAATGACCTTCCT-3' and reverse, 5'-TGGCTGAATGCTTCTTGGGT-3'; miR-9a-5p forward, 5'-GCGGCGGTCTTTGGTTATCTAG-3' and reverse, 5'-ATCCAGTGCAGGGTCCGAGG-3'; and BCL2L11 forward, 5'-CACCAGCACCATAGAAGAA-3' and reverse, 5'-ATAAGGAGCAGGCACAGA-3'. The following thermocycling conditions were used for qPCR: Initial denaturation for $5 \mathrm{~min}$ at $95^{\circ} \mathrm{C}$; followed by 35 cycles of $95^{\circ} \mathrm{C}$ for $10 \mathrm{sec}$ and $60^{\circ} \mathrm{C}$ for $30 \mathrm{sec}$. U6 or GAPDH were used as the internal controls. The relative mRNA expression levels of lncRNA TUG1, miR-9a-5p and BCL2L11 were calculated using the $2^{-\Delta \Delta \mathrm{Cq}}$ method (24). All samples were analyzed at least in triplicate.

miRNA target analysis and dual luciferase reporter assay. The association between miR-9a-5p and BCL2L11 was identified using TargetScan version 7.1 (targetscan.org/vert_71). The 3'-untranslated region products of BCL2L11 containing the target sequence of miR-9a-5p were obtained by RT-qPCR and fused into a pmirGLO vector (Promega Corporation) to construct the BCL2L11-wild-type (BCL2L11-WT) and BCL2L11-mutated-type (BCL2L11-MUT) reporter vectors. The results showed that BCL2L11 is a potential target of miR-9a-5p. 293 cells (ATCC ${ }^{\circledR}$ CRL-1573; ATCC), that were cultured $>24 \mathrm{~h}$, were transfected with BCL2L11-WT or 
A

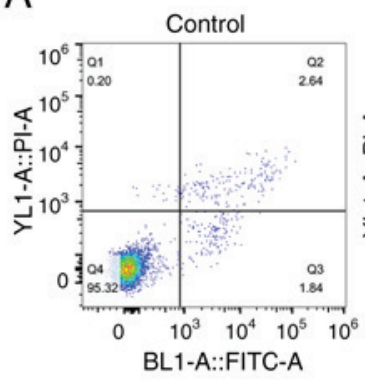

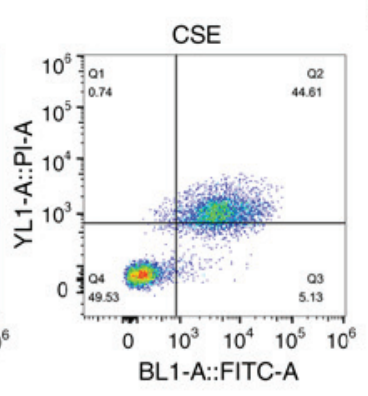

B

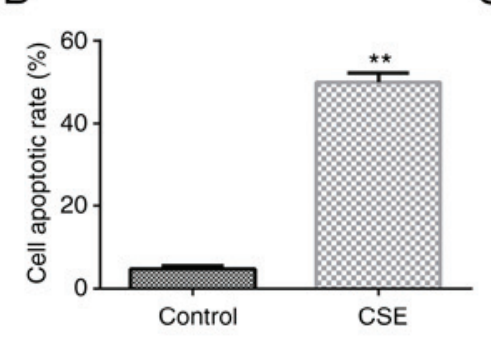

C

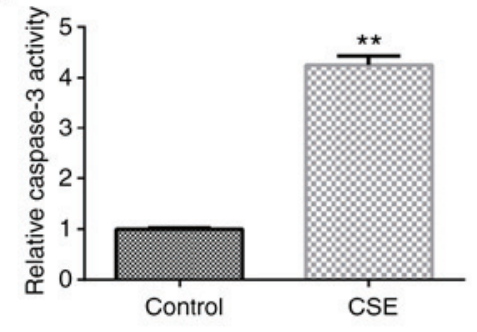

D

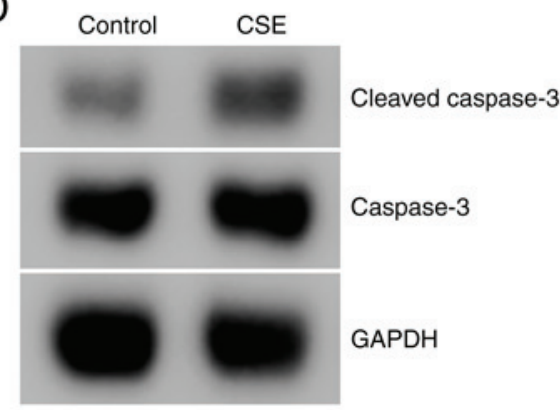

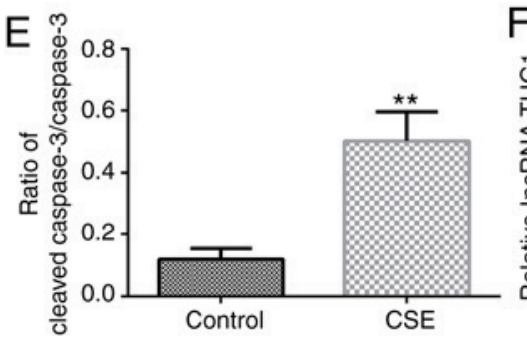

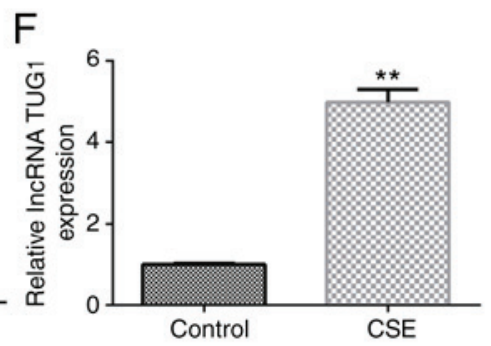

Figure 1. CSE affects HPMEC apoptosis. (A and B) Cell apoptotic rates of HPMECs were detected using flow cytometry. (C) Caspase-3 activity in HPMECs following CSE treatment was detected using a Caspase-3 Activity assay kit. (D) Western blotting was performed to detect the relative protein expression of cleaved Caspase-3 and Caspase-3 in HPMECs. (E) Ratio of cleaved Caspase-3/Caspase-3. (F) Expression levels of lncRNA TUG1 in HPMECs were measured by reverse transcription-quantitative PCR analysis. ${ }^{* *} \mathrm{P}<0.01$ vs. Control. Experiments were repeated three times. An unpaired Student's t-test was used to analyze the data. CSE, cigarette smoke extract; HPMEC, human pulmonary microvascular endothelial cell; lncRNA TUG1, long non-coding RNA taurine-upregulated gene 1.

BCL2L11-MUT and miR-9a-5p mimic or mimic control using Lipofectamine ${ }^{\circledR} 2000$ reagent (Invitrogen; Thermo Fisher Scientific, Inc.) for $48 \mathrm{~h}$. Luciferase activity was measured using a dual luciferase reporter assay system (Promega Corporation) according to the manufacturer's instructions. Renilla luciferase activity was used as a control. All the experiments were performed at least three times.

Cell transfection. Normal HPMECs were inoculated in 6-well plates overnight and transfected with control-small interfering RNA (siRNA), TUG1-siRNA, inhibitor control, miR-9a-5p inhibitor, TUG1-siRNA + inhibitor control, TUG1-siRNA + miR-9a-5p inhibitor, mimic control, miR-9a-5p mimic, control-plasmid, BCL2L11-plasmid, miR-9a-5p mimic + control-plasmid or miR-9a-5p mimic + BCL2L11-plasmid (Shanghai GenePharma Co., Ltd.) using Lipofectamine ${ }^{\circledR} 2000$ reagent (Invitrogen; Thermo Fisher Scientific, Inc.). A total of $48 \mathrm{~h}$ after transfection, cells were collected, and the transfection efficiency was assessed using RT-qPCR. For the in vitro analysis, $48 \mathrm{~h}$ after cell transfection, the cells were subjected to $1 \%$ CSE for $24 \mathrm{~h}$. Cells without any treatment were used as the control.

Caspase-3 activity assay. The activities of caspase-3 were measured using a colorimetric assay kit (cat. no. C1115; Beyotime Institute of Biotechnology) in accordance with the manufacturer's instructions.

Statistical analysis. Data are presented as the mean \pm standard deviation of at least three independent tests. Statistical comparisons between groups were assessed using a Student's t-test or a one-way ANOVA followed by a Tukey's post hoc test. $\mathrm{P}<0.05$ was considered to indicate a statistically significant difference.

\section{Results}

CSE influences HPMEC growth. Cell apoptosis of HPMECs treated with $1 \% \mathrm{CSE}$ for $24 \mathrm{~h}$ was detected by FCM. The results showed that CSE significantly induced cell apoptosis in HPMECs (Fig. 1A and B). Compared with the control group, 1\% CSE markedly increased caspase-3 activity, cleaved caspase- 3 protein expression and the cleaved-caspase-3/caspase-3 ratio (Fig. 1C-E). Moreover, lncRNA TUG1 expression in HPMECs was significantly increased with $1 \%$ CSE treatment compared with the control group (Fig. 1F). The results confirmed that lncRNA TUG1 was significantly upregulated in $1 \%$ CSE induced HPMECs.

TUG1 negatively regulates miR-9a-5p expression in HPMECs. A previous study showed the presence of a binding site between TUG1 and miR-9a-5p (25) (Fig. 2A). In the present study, the targeted relationship between TUG1 and miR-9a-5p was verified using a dual luciferase reporter assay (Fig. 2B). Moreover, miR-9a-5p expression in HPMECs was significantly decreased following the $1 \%$ CSE treatment compared with the control group (Fig. 2C). To further assess the regulatory relationship between TUG1 and miR-9a-5p, HPMECs were transfected with different plasmids, and the transfection efficiency was determined using RT-qPCR. As shown in Fig. 2D and E, TUG1-siRNA and miR-9a-5p inhibitor significantly reduced the expression 
A Target: $5^{\prime}$ agAUGACGACUUGAUUACCAAAGa $3^{\prime}$

|| ||: || ||| |||||||

miRNA: $3^{\prime}$ agUA-UGUCGAUCUAUUGGUUUCU $5^{\prime}$
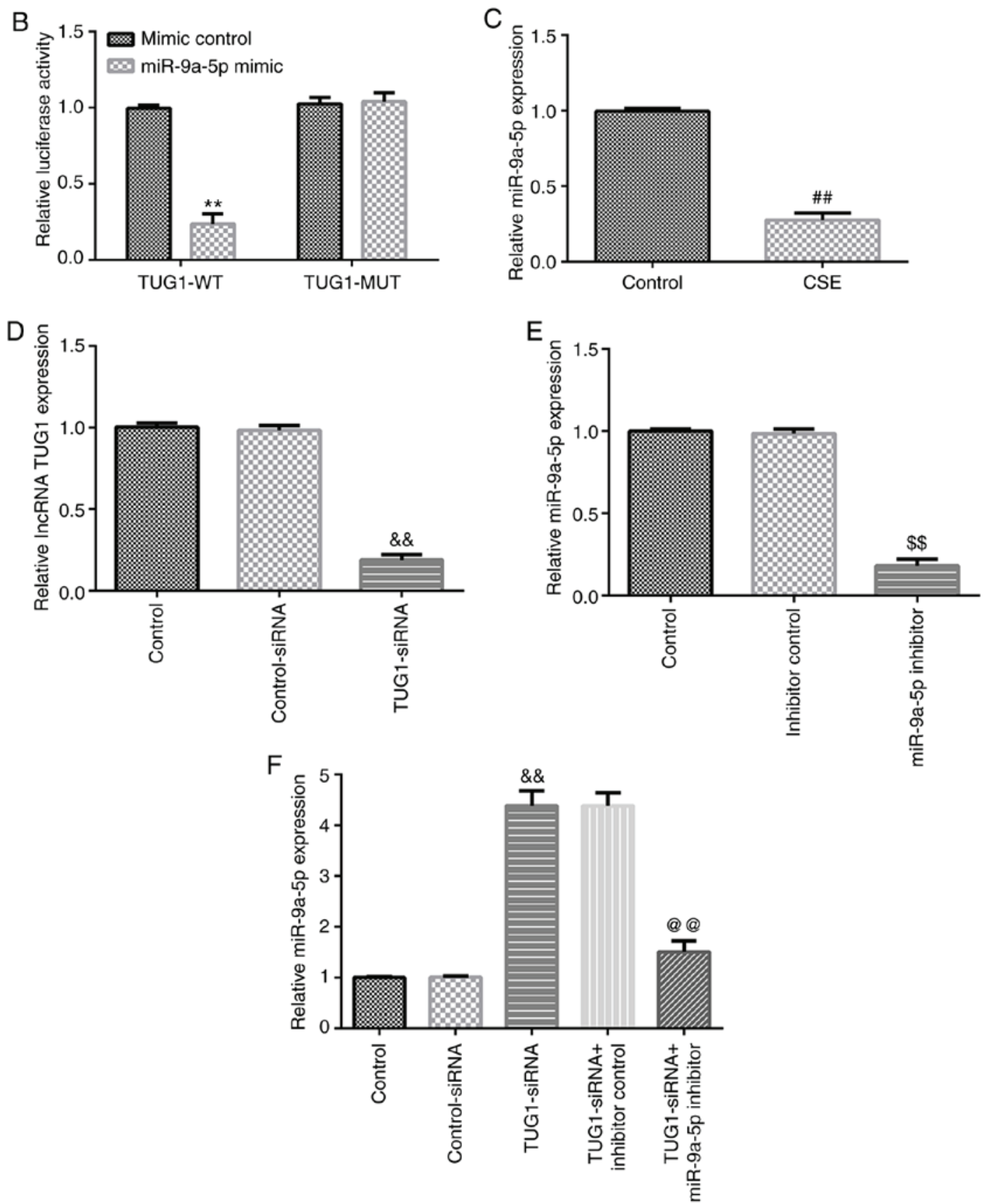

Figure 2. TUG1 negatively regulates miR-9a-5p in HPMECs. (A) The association between TUG1 and miR-9a-5p was predicted using bioinformatics software. (B) The association between TUGland miR-9a-5p was verified using a dual luciferase reporter assay. (C) Levels of miR-9a-5p in HPMECs following CSE treatment were detected using RT-qPCR. (D-F) RT-qPCR was used to quantify the mRNA expression levels of TUG1 and miR-9a-5p in transfected HPMECs. Experiments were repeated three times. A one-way ANOVA with a post-hoc Tukey's test or an unpaired Student's t-test were used for analysis. ${ }^{* *} \mathrm{P}<0.01$ vs. mimic control; ${ }^{\# \#} \mathrm{P}<0.01$ vs. Control; ${ }^{\& \&} \mathrm{P}<0.01$ vs. Control-siRNA; ${ }^{\$ \$} \mathrm{P}<0.01$ vs. inhibitor control; ${ }^{\circledR} \mathrm{P}<0.01$ vs. TUG1-siRNA+inhibitor control. TUG1, taurine-upregulated gene 1; miR, microRNA; CSE, cigarette smoke extract; HPMEC, human pulmonary microvascular endothelial cells; RT-qPCR, reverse transcription-quantitative PCR.

of TUG1 and miR-9a-5p, respectively. Compared with the control-siRNA group, TUG1-siRNA significantly increased miR-9a-5p expression in HPMECs, which was reversed by co-transfection with miR-9a-5p inhibitor (Fig. 2F). The data indicated that TUG1 negatively regulated miR-9a-5p expression in HPMECs.
TUG1 inhibits cell apoptosis induced by CSE in HPMECs by regulating miR-9a-5p. As shown in Fig. 3A-F, 1\% CSE significantly induced cell apoptosis, increased caspase-3 activity, enhanced cleaved caspase- 3 protein expression, increased the cleaved caspase-3/caspase- 3 ratio and downregulated miR-9a-5p expression in HPMECs. Moreover, compared with 

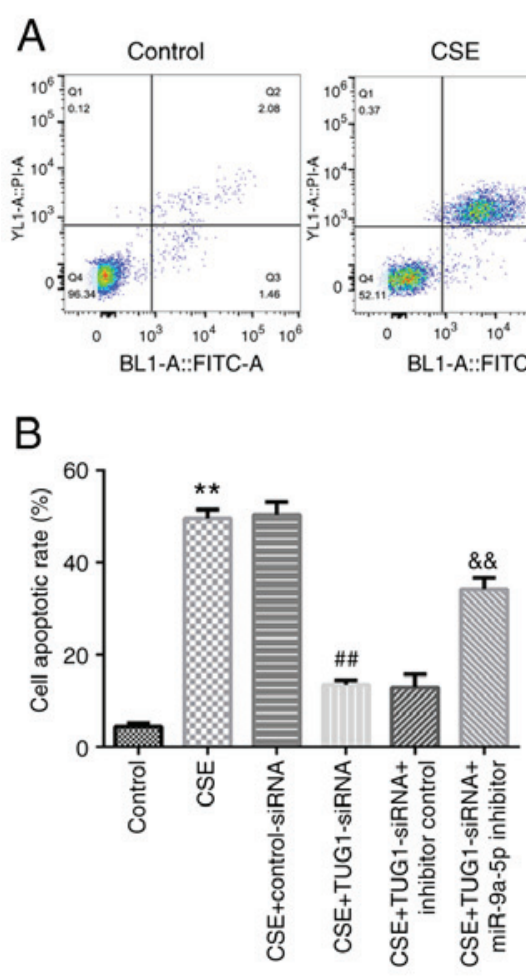

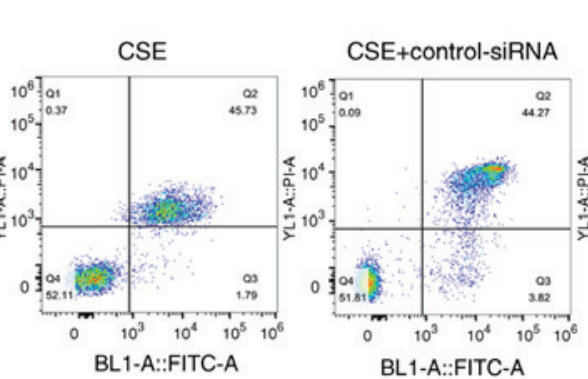

$\mathrm{C}$

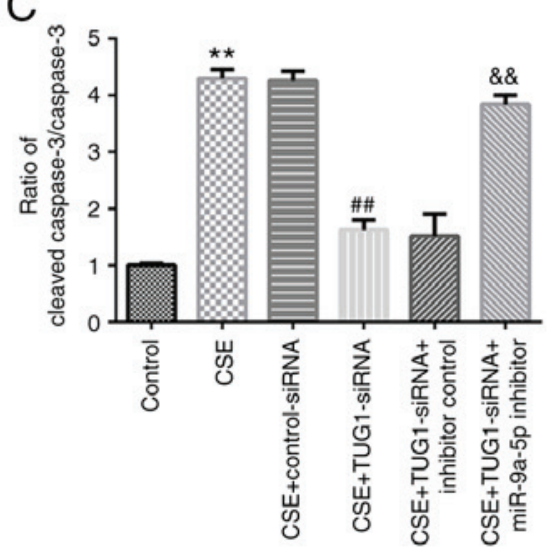

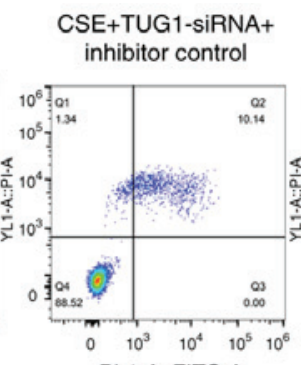

CSE+TUG1-siRNA+ miR-9a-5p inhibitor

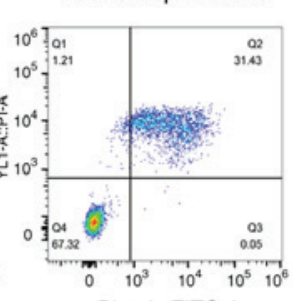

D

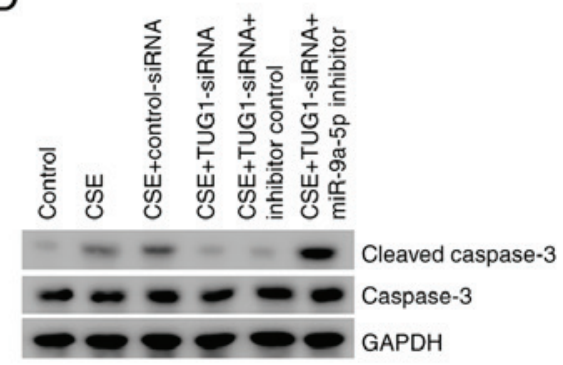

E

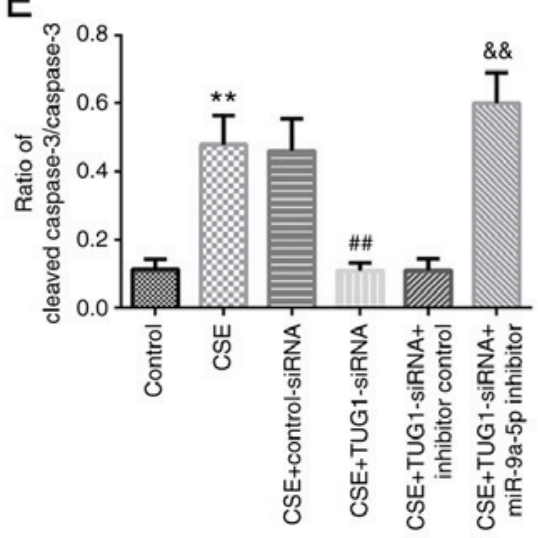

$\mathrm{F}$

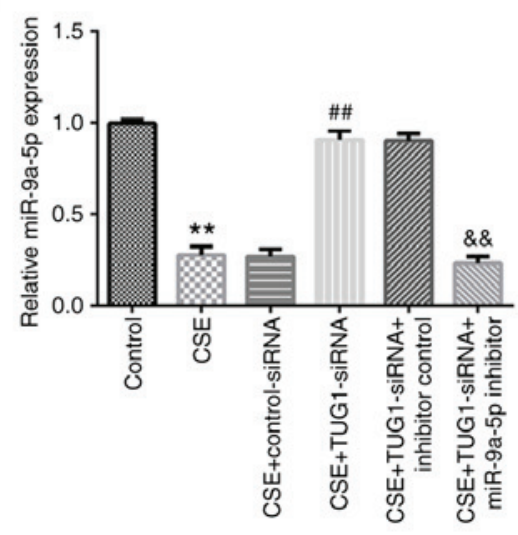

Figure 3. TUG1 affects cell apoptosis induced by CSE in HPMECs by regulating miR-9a-5p. (A and B) Apoptotic rates of HPMECs were detected using flow cytometry analysis. (C) Caspase-3 activity in HPMECs was detected using a Caspase-3 Activity assay kit. (D) Western blot analysis was used to detect the relative protein expression of cleaved Caspase-3 and Caspase-3 in HPMECs. (E) The ratio of cleaved-Caspase-3/Caspase-3. (F) miR-9a-5p expression in HPMECs were measured by reverse transcription-quantitative PCR analysis. Experiments were repeated three times. A one-way ANOVA with a post-hoc Tukey's test was used for analysis. ${ }^{* *} \mathrm{P}<0.01$ vs. Control; ${ }^{\# /} \mathrm{P}<0.01$ vs. CSE+control-siRNA; \&\& $\mathrm{P}<0.01$ vs. CSE+TUG1-siRNA+inhibitor control. TUG1, taurine-upregulated gene 1; miR, microRNA; CSE, cigarette smoke extract; HPMEC, human pulmonary microvascular endothelial cell.

the CSE + control-siRNA group, TUG1-siRNA significantly reduced cell apoptosis, caspase- 3 activity, cleaved caspase-3 protein expression and the cleaved caspase-3/caspase-3 ratio, and upregulated miR-9a-5p expression in HPMECs. All these changes were reversed by miR-9a-5p inhibitor co-transfection. The above results suggested that TUG1 inhibited cell apoptosis induced by CSE in HPMECs by regulating miR-9a-5p.

BCL2L11 is a direct target gene of miR-9a-5p. TargetScan analysis showed the presence of a binding site between BCL2L11 and miR-9a-5p (Fig. 4A), which was verified by a dual luciferase reporter assay (Fig. 4B). Moreover, the mRNA expression of BCL2L11 in HPMECs was significantly increased with $1 \%$ CSE treatment compared with the control group (Fig. 4C). Further results showed that miR-9a-5p expression in the miR-9a-5p mimic group was higher compared with the mimic control group (Fig. 4D). BCL2L11-plasmid significantly increased BCL2L11 mRNA expression in HPMECs (Fig. 4E). Compared with the mimic control group, miR-9a-5p mimic significantly decreased BCL2L11 mRNA expression in HPMECs, which was reversed by the co-transfection with the BCL2L11-plasmid (Fig. 4F). Thus, it was shown that BCL2L11 was the direct target gene of miR-9a-5p, and it was negatively regulated by miR-9a-5p.

miR-9a-5p inhibits cell apoptosis induced by CSE in HPMECs by regulating BCL2L11. Cells were treated 


\section{A}

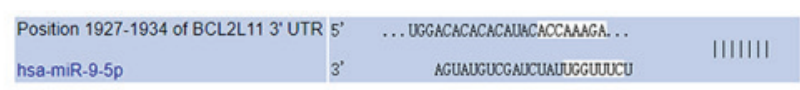

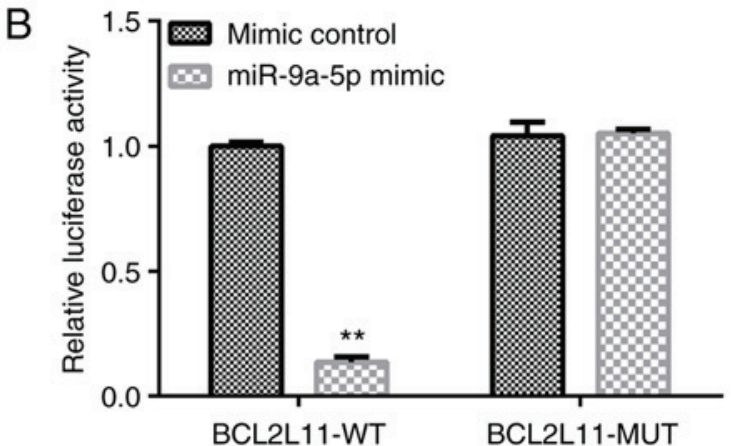
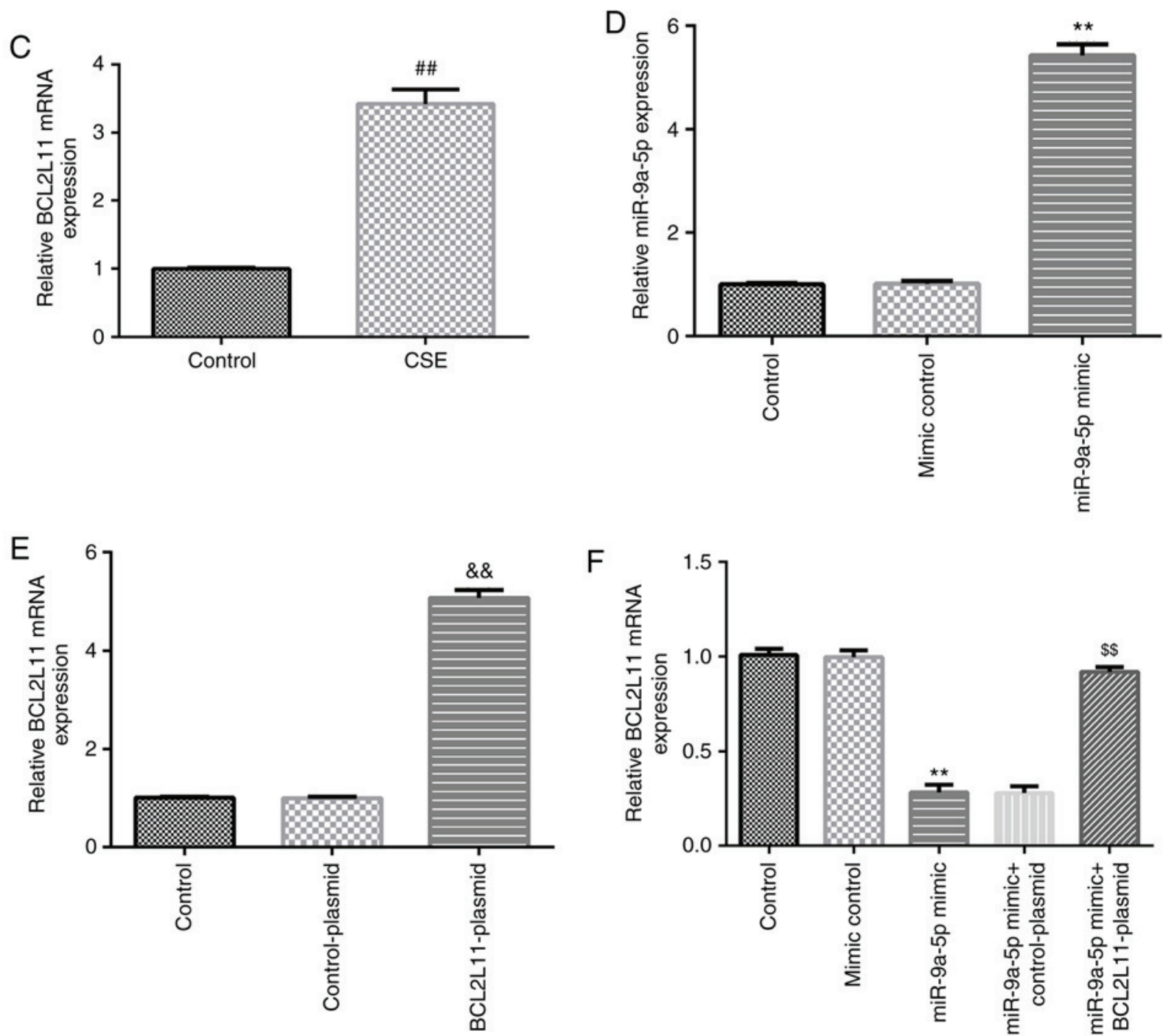

$\mathrm{F}$

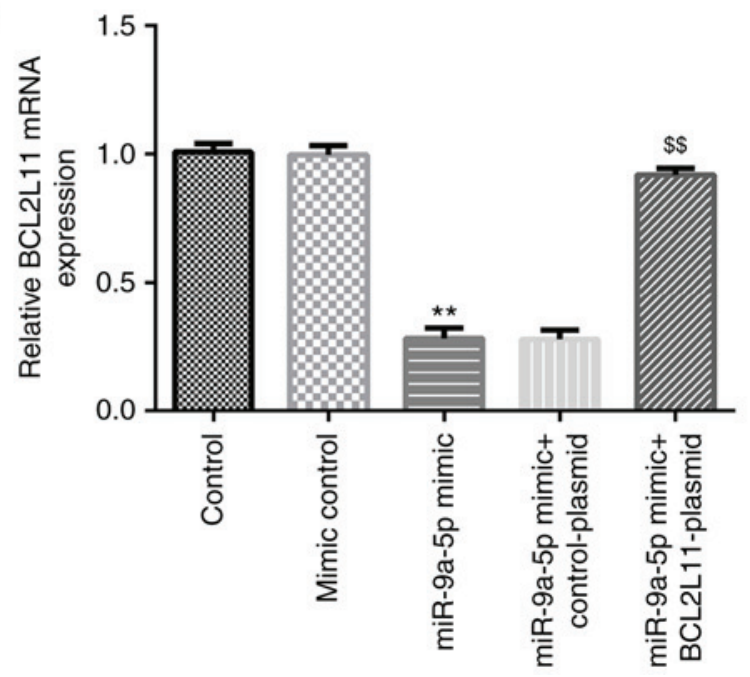

Figure 4. miR-9a-5p directly targets BCL2L11. (A) Predicted wild-type miR-9a-5p binding sites in the 3'-UTR of BCL2L11. (B) Dual-luciferase reporter assays were performed to verify the binding sites between miR-9a-5p and BCL2L11. (C) The mRNA levels of BCL2L11 in HPMECs following CSE treatment were detected using RT-qPCR. (D-F) RT-qPCR was performed to quantify the mRNA expression levels of miR-9a-5p and BCL2L11 in transfected HPMECs. Experiments were repeated three times. A one-way ANOVA with a post-hoc Tukey's test or an unpaired Student's t-test were used for analysis. ${ }^{* *} \mathrm{P}<0.01 \mathrm{vs}$ mimic control; ${ }^{\# \#} \mathrm{P}<0.01$ vs. Control; \&\& $\mathrm{P}<0.01$ vs. Control-plasmid; ${ }^{\$ \$} \mathrm{P}<0.01$ vs.miR-9a-5p mimic +control-plasmid. miR, microRNA; CSE, cigarette smoke extract; HPMEC, human pulmonary microvascular endothelial cell; UTR, untranslated region; RT-qPCR, reverse transcription-quantitative PCR; BCL2L11, Bcl-2 like 11.

with $1 \%$ CSE following transfection. Cell apoptosis, caspase- 3 activity, cleaved caspase- 3 expression, the cleaved caspase-3/caspase-3 ratio and BCL2L11 expression were detected. The results showed that CSE significantly induced cell apoptosis, increased caspase-3 activity, cleaved caspase-3 expression and the cleaved caspase-3/caspase-3 ratio, and enhanced BCL2L11 protein and mRNA expression in HPMECs. Similarly, compared with the CSE + mimic control group, miR-9a-5p mimic inhibited cell apoptosis, decreased caspase- 3 activity, cleaved caspase- 3 expression and the 

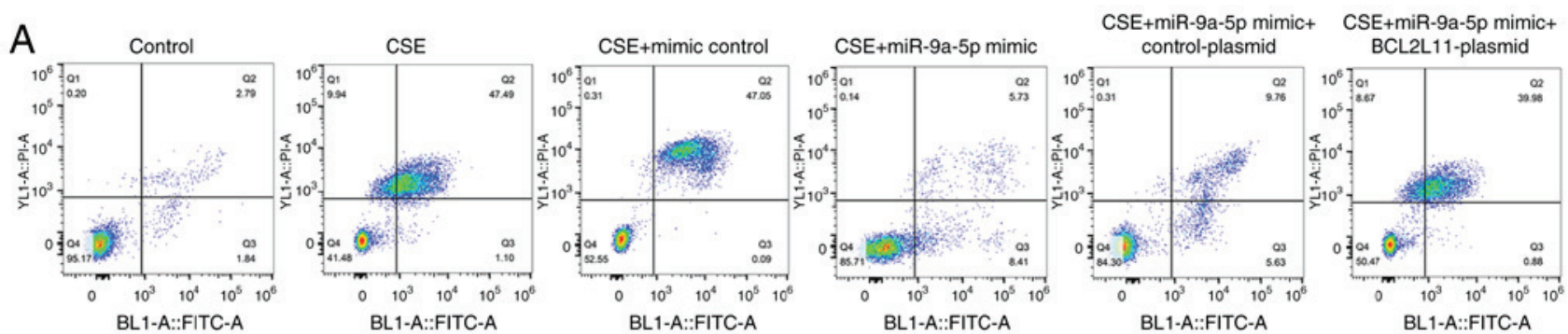

BL1-A::FITC-A

BL1-A::FITC-A

BL1-A:FITC-A

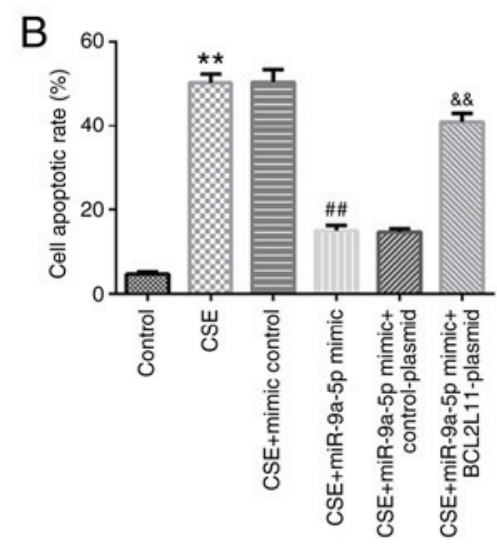

E

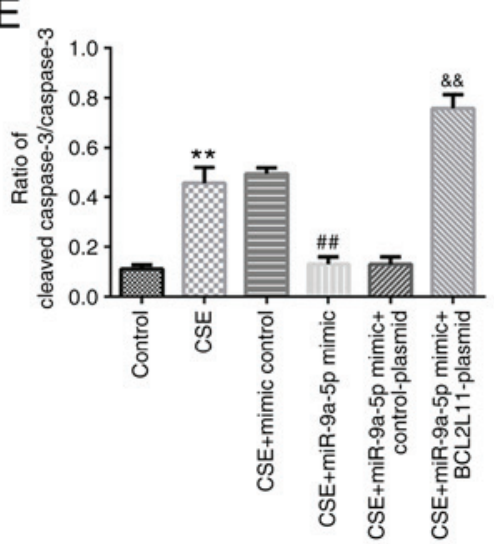

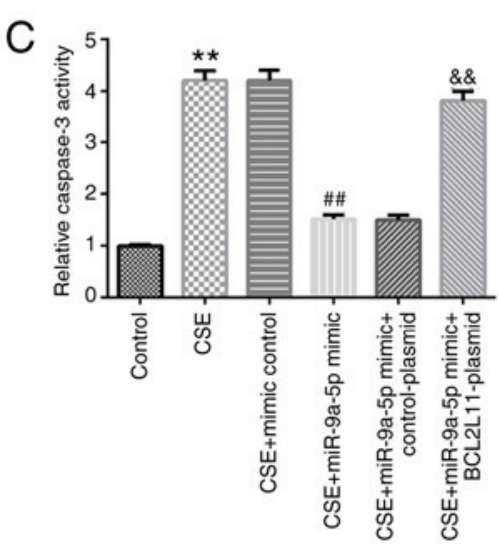

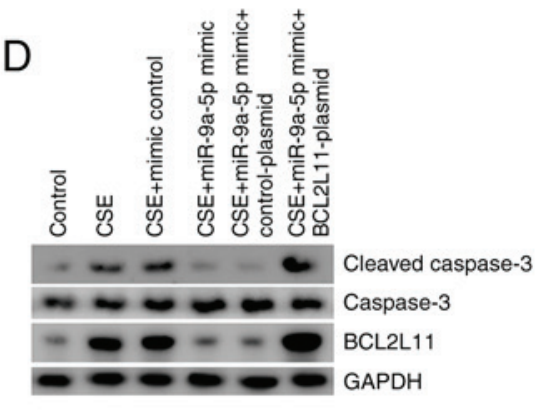

Figure 5. miR-9a-5p affects HPMEC cell apoptosis induced by CSE via regulating BCL2L11 expression. (A and B) Apoptotic rates of HPMECs were detected by flow cytometry analysis. (C) Caspase-3 activity in HPMECs was detected using a Caspase-3 Activity assay kit. (D) Western blot analysis was performed to detect the relative protein expression levels of cleaved Caspase-3, Caspase-3 and BCL2L11 in HPMECs. (E) The ratio of cleaved Caspase-3/Caspase-3. (F) mRNA expression levels of BCL2L11 in HPMECs were measured using reverse transcription-quantitative PCR analysis. Experiments were repeated three times. A one-way ANOVA with a post-hoc Tukey's test or an unpaired Student's t-test were used for analysis. ${ }^{* *} \mathrm{P}<0.01 \mathrm{vs}$. Control; ${ }^{\# \#} \mathrm{P}<0.01 \mathrm{vs}$. CSE+mimic control; ${ }^{\&} \mathrm{P}<0.01$ vs. CSE+miR-9a-5p mimic+control-plasmid. miR, microRNA; CSE, cigarette smoke extract; HPMEC, human pulmonary microvascular endothelial cell; BCL2L11, Bcl-2 like 11.

cleaved caspase-3/caspase-3 ratio, and reduced BCL2L11 protein and mRNA expression in HPMECs. These changes were significantly reversed by BCL2L11-plasmid co-transfection (Fig. 5A-F). In conclusion, miR-9a-5p inhibited cell apoptosis induced by CSE in HPMECs by regulating BCL2L11.

\section{Discussion}

COPD is a chronic lung disease characterized by incomplete reversible airflow limitation. Common complications include chronic respiratory failure, spontaneous pneumothorax and chronic pulmonary heart disease $(4,26)$. The global incidence of COPD amongst individuals $>40$ years of age is $9-10 \%$. COPD not only causes respiratory impairment, but also affects circulatory function and can be life-threatening in severe cases (27). HPMEC growth is associated with the process of COPD. HPMECs were used in the present study, and it was found that $1 \%$ CSE induced cell apoptosis and increased lncRNA TUG1 expression in HPMECs, consistent with previous reports. The results showed that increased lncRNA TUG1 levels could be used as a diagnostic marker for COPD.

Furthermore, the present study investigated the mechanisms of lncRNA TUG1 in COPD. It was previously reported that there is a binding site between TUG1 and miR-9a-5p (25), 
and this association was verified in the present study. In addition, a negative regulatory mechanism was found between lncRNA TUG1 and miR-9a-5p in HPMECs. Endothelial apoptosis serves a key role in the pathogenesis of COPD $(13,14)$. The lncRNA TUG1/miR-9a-5p axis has also been reported to serve a significant role in the regulation of cell apoptosis (25). Thus, it was hypothesized that TUG1 may be involved in COPD pathogenesis through regulating EC apoptosis. Therefore, whether TUG1 affected cell apoptosis in HPMECs via regulation of miR-9a-5p was determined, and several experiments in HPMECs were performed following knockdown of TUG1 or miR-9a-5p expression. FCM analysis indicated that knocking down TUG1 reduced cell apoptosis induced by CSE in HPMECs, and the effects were reversed by downregulating miR-9a-5p. Similarly, the present study predicted and verified that BCL2L11 is the direct target gene of miR-9a-5p. miR-9a-5p was found to negatively regulate BCL2L11 expression in HPMECs. BCL2L11, a member of the BCL-2 family, is located in the outer membrane of mitochondria and it serves as an important regulator of apoptosis $(28,29)$. Thus, miR-9a-5p may regulate HPMECs apoptosis via negatively regulating BCL2L11 expression. The FCM results showed that miR-9a-5p overexpression relieved cell apoptosis induced by CSE in HPMECs, and the effect was reversed by upregulating BCL2L11. The caspase family participates in mediating cell apoptosis, and caspase- 3 is a key regulatory molecule involved in numerous pathways in apoptotic signal transduction $(30,31)$. The present study detected caspase- 3 activity and expression. The results indicated that TUG1, miR-9a-5p and BCL2L11 affected HPMEC apoptosis by regulating caspase-3.

In conclusion, the present study found that lncRNA TUG1 serves a role in cell apoptosis induced by CSE in HPMECs via modulating a miR-9a-5p/BCL2L11 axis; the mechanism identified in the present study is shown in Fig. S1. These findings suggested that TUG1 was a potential effective target for COPD treatment.

\section{Acknowledgements}

Not applicable.

\section{Funding}

No funding was received.

\section{Availability of data and materials}

The datasets used and/or analyzed during the current study are available from the corresponding author on reasonable request.

\section{Authors' contributions}

$\mathrm{XC}$ and MM conceived and designed the current study, as well as acquired, analyzed and interpreted the data, and prepared the manuscript. YS and XJ contributed to the acquisition and analysis of the data. ZY contributed to acquisition and analysis of the data, and prepared the manuscript. All authors read and approved the final manuscript, and confirm the authenticity of all the raw data.

\section{Ethics approval and consent to participate}

Not applicable.

\section{Patient consent for publication}

Not applicable.

\section{Competing interests}

The authors declare that they have no competing interests.

\section{References}

1. Rabe KF and Watz H: Chronic obstructive pulmonary disease. Lancet 389: 1931-1940, 2017.

2. Duffy SP and Criner GJ: Chronic obstructive pulmonary disease: Evaluation and management. Med Clin North Am 103: 453-461,2019.

3. Lareau SC, Fahy B, Meek P and Wang A: Chronic obstructive pulmonary disease (COPD). Am J Respir Crit Care Med 199: P1-P2, 2019.

4. Berg K and Wright JL: The pathology of chronic obstructive pulmonary disease: Progress in the 20th and 21st centuries. Arch Pathol Lab Med 140: 1423-1428, 2016.

5. Sorathia L: Palliative care in chronic obstructive pulmonary disease. Med Clin North Am 103: 517-526, 2019.

6. van Geffen WH, Kerstjens HAM and Slebos DJ: Emerging bronchoscopic treatments for chronic obstructive pulmonary disease. Pharmacol Ther 179: 96-101, 2017.

7. Aghapour M, Raee P, Moghaddam SJ, Hiemstra PS and Heijink IH: Airway epithelial barrier dysfunction in chronic obstructive pulmonary disease: Role of cigarette smoke exposure. Am J Respir Cell Mol Biol 58: 157-169, 2018.

8. Postma DS, Bush A and van den Berge M: Risk factors and early origins of chronic obstructive pulmonary disease. Lancet 385: 899-909, 2015.

9. Barnes PJ: Inflammatory mechanisms in patients with chronic obstructive pulmonary disease. J Allergy Clin Immunol 138: 16-27, 2016.

10. Sohal SS: Epithelial and endothelial cell plasticity in chronic obstructive pulmonary disease (COPD). Respir Investig 55: 104-113, 2017.

11. Butt Y,Kurdowska A and Allen TC: Acute lung injury: A clinical and molecular review. Arch Pathol Lab Med 140: 345-350, 2016.

12. Siganaki M, Koutsopoulos AV, Neofytou E, Vlachaki E, Psarrou M, Soulitzis N, Pentilas N, Schiza S, Siafakas NM and Tzortzaki EG: Deregulation of apoptosis mediators' p53 and bcl2 in lung tissue of COPD patients. Respir Res 11: 46, 2010.

13. Chen Y, Luo H, Kang N, Guan C, Long Y, Cao J, Shen Q, Li J, Yang $\mathrm{M}$, Peng $\mathrm{H}$ and Chen P: Beraprost sodium attenuates cigarette smoke extract-induced apoptosis in vascular endothelial cells. Mol Biol Rep 39: 10447-10457, 2012.

14. Yang M, Chen P, Peng H, Zhang H, Chen Y, Cai S, Lu Q and Guan C: Cigarette smoke extract induces aberrant cytochrome-c oxidase subunit II methylation and apoptosis in human umbilical vascular endothelial cells. Am J Physiol Cell Physiol 308: C378-C384, 2015.

15. Sun Y, An N, Li J, Xia J, Tian Y, Zhao P, Liu X, Huang H, Gao J and Zhang X: miRNA-206 regulates human pulmonary microvascular endothelial cell apoptosis via targeting in chronic obstructive pulmonary disease. J Cell Biochem 120: 6223-6236, 2019.

16. Hombach S and Kretz M: Non-coding RNAs: Classification, biology and functioning. Adv Exp Med Biol 937: 3-17, 2016.

17. Novak J, Vašků JB and Souček M: Long non-coding RNAs in the pathophysiology of atherosclerosis. Vnitr Lek 64: 77-82, 2018 (In Czech).

18. Beermann J, Piccoli MT, Viereck J and Thum T: Non-coding RNAs in development and disease: Background, mechanisms, and therapeutic approaches. Physiol Rev 96: 1297-1325, 2016.

19. Kok FO and Baker AH: The function of long non-coding RNAs in vascular biology and disease. Vascul Pharmacol 114: 23-30, 2019.

20. Zhang H, Sun D, Li D, Zheng Z, Xu J, Liang X, Zhang C, Wang S, Wang $\mathrm{J}$ and Lu W: Long non-coding RNA expression patterns in lung tissues of chronic cigarette smoke induced COPD mouse model. Sci Rep 8: 7609, 2018. 
21. Tang W, Shen Z, Guo J and Sun S: Screening of long non-coding RNA and TUG1 inhibits proliferation with TGF- $\beta$ induction in patients with COPD. Int J Chron Obstruct Pulmon Dis 11: 2951-2964, 2016.

22. Gu W, Yuan Y, Wang L, Yang H, Li S, Tang Z and Li Q: Long non-coding RNA TUG1 promotes airway remodelling by suppressing the miR-145-5p/DUSP6 axis in cigarette smoke-induced COPD. J Cell Mol Med 23: 7200-7209, 2019.

23. Li D, Hu J, Wang T, Zhang X, Liu L, Wang H, Wu Y, Xu D and Wen F: Silymarin attenuates cigarette smoke extract-induced inflammation via simultaneous inhibition of autophagy and ERK/p38 MAPK pathway in human bronchial epithelial cells. Sci Rep 6: 37751, 2016.

24. Livak KJ and Schmittgen TD: Analysis of relative gene expression data using real-time quantitative PCR and the 2(-Delta Delta C(T)) method. Methods 25: 402-408, 2001.

25. Yang D, Yu J, Liu HB, Yan XQ, Hu J, Yu Y, Guo J, Yuan Y and Du ZM: The long non-coding RNA TUG1-miR-9a-5p axis contributes to ischemic injuries by promoting cardiomyocyte apoptosis via targeting KLF5. Cell Death Dis 10: 908, 2019.

26. Riley CM and Sciurba FC: Diagnosis and outpatient management of chronic obstructive pulmonarydisease: A review. JAMA 321: 786-797, 2019.

27. Lin YH, Tsai CL, Tsao LI and Jeng C: Acute exacerbations of chronic obstructive pulmonary disease (COPD) experiences among COPD patients with comorbid gastrooesophageal reflux disease. J Clin Nurs 28: 1925-1935, 2019.
28. Concannon CG, Tuffy LP, Weisová P, Bonner HP, Dávila D, Bonner C, Devocelle MC, Strasser A, Ward MW and Prehn JH: AMP kinase-mediated activation of the $\mathrm{BH} 3$-only protein Bim couples energy depletion to stress-induced apoptosis. J Cell Biol 189: 83-94, 2010

29. Kilbride SM, Farrelly AM, Bonner C, Ward MW, Nyhan KC, Concannon CG, Wollheim CB, Byrne MM and Prehn JH: AMP-activated protein kinase mediates apoptosis in response to bioenergetic stress through activation of the pro-apoptotic Bcl-2 homology domain-3-only protein BMF. J Biol Chem 285: 36199-36206, 2010.

30. Crowley LC and Waterhouse NJ: Detecting cleaved caspase-3 in apoptotic cells by flow cytometry. Cold Spring Harb Protoc: Nov 1, 2016 (Epub ahead of print). doi: 10.1101/pdb.prot087312.

31. Rogers C, Fernandes-Alnemri T, Mayes L, Alnemri D, Cingolani $G$ and Alnemri ES: Cleavage of DFNA5 by caspase-3 during apoptosis mediates progression to secondary necrotic/pyroptotic cell death. Nat Commun 8: 14128, 2017.

This work is licensed under a Creative Commons Attribution-NonCommercial-NoDerivatives 4.0 International (CC BY-NC-ND 4.0) License. 